\title{
Breathe, Relax and Remember: An investigation into how Focused Breathing can improve identification of EvoFIT facial composites
}

\author{
Alexander J. Martin ${ }^{1}$, Peter J. B. Hancock ${ }^{1}$ and Charlie D. Frowd ${ }^{2}$ \\ ${ }^{1}$ Psychology, Faculty of Natural Sciences, University of Stirling, Stirling, UK \\ ${ }^{2}$ Department of Psychology, University of Central Lancashire, Preston, UK \\ ajm00027@students.stir.ac.ukp.j.b.hancock@stir.ac.uk cfrowd1@uclan.ac.uk
}

\begin{abstract}
Accurate identification of criminal suspects by witnesses is vital for police investigations. Methods such as Cognitive Interviewing techniques have been employed for this reason to enhance witness recall. In the current project, we demonstrate the benefit of including a focused breathing exercise during face construction using the EvoFIT recognition-based facial composite system. Twenty participants, half of whom received the focused breathing instruction, each constructed a facial composite of an unfamiliar face seen the previous day. A further 40 participants attempted to name the resulting composites. $A$ significant increase was found in accurate identification of composites constructed by the focused breathing group.

Keywords: EvoFIT; focused breathing; interview; familiar and unfamiliar face recognition; witness; victim.
\end{abstract}

\section{Introduction}

Offenders often leave no physical (e.g., fingerprints, DNA samples) or photographic (e.g., CCTV images) evidence at a crime scene [1]. However, when available, police may ask an eyewitness or a victim (who is sometimes the same person) to complete a facial composite of the offender [2]. A facial composite is typically created by a witness who describes the offender in detail and is then guided through the construction process by a forensic practitioner [3]. The resulting composite may then be shown to a wider audience (e.g., media outlets such as newspapers and television news programmes) in the hope a member of the public recognises it and an arrest of the offender can be made [2]. Research has established that the effectiveness of a composite can be affected by a number factors including the composite system, the interviewing techniques administered by the practitioner [5], the method used to present the composite to the media [3], and factors such as anxiety that may involve the witness him or herself [6].

Several composite systems have been employed throughout the past 50 years or so. Their effectiveness has been variable [1]. The most successful of these (EvoFIT) achieved accurate suspect identifications of around 30\% [7] in early implementations, but around 60\% more recently [2]. Additionally, modern interviewing techniques have been devised to help improve a witness's recollection of the offender's face. Cognitive interviewing invites the witness to recall as much detail about the crime scene and the offender as possible [8], and holistic style interviewing focuses on personality traits that the witness may have perceived from the offender's face [9]. Combined with EvoFIT, these procedures have been found to be valuable for identifying suspects (e.g., [7]).

Whilst showing some efficacy for recall [2], techniques such as these do little to address other factors which may be detrimental to recall, such as anxiety and stress levels that may be experienced by witnesses [10]. Some stress may be considered necessary, and even desirable for mundane tasks (e.g., getting out of bed in the morning). However, too much stress can be detrimental to physical and mental wellbeing, particularly when brought about by excessive cognitive anxiety [11]. As cognitive resources may be limited [12], competition between anxiety and recall may well affect a witness's ability to access memory for accurate details of a crime [13]. For many witnesses, attending a police interview may be a novel experience, potentially laden with anxiety and stress [6]. Witnesses may be traumatised or simply anxious about attending a police station [11]. Consequently, the interview may be conducted under less than optimal conditions. While some aspects of an investigation are not under control of the criminal justice system (e.g., when a crime takes place, the appearance of the criminal), it should be possible to lessen a witness's level of anxiety once they are part of the criminal justice system.

Here, we employed existing EvoFIT procedures [14] and add a novel focused breathing technique designed specifically to address issues with anxiety. Based on mindfulness training, focused breathing is designed to relax a person, the result of which should enhance recall [18,31,32]. Mindfulness has its roots in meditative techniques, commonly ascribed to Buddhist practices [15]. Much of the research on mindfulness has been conducted in clinical settings and usually takes the form of meditation training that can take several weeks to complete [16]. It takes many forms (e.g., meditation, focused breathing, attentional control) but can be described as having a fully conscious awareness of the present moment [17]. Mindfulness can be 
beneficial in practical areas of psychology including memory retrieval [18], depression [19] and anxiety [20].

Unfortunately, even these apparent benefits do not appear to translate well to techniques that would facilitate facial composite accuracy, as it would be impractical for police to send witnesses on a mindfulness training course. However, some research has considered shorter mindfulness training time-scales. Arch and Craske (2006) [21] looked at the effect a 15-minute focused breathing exercise may have on regulation of emotions, and found recipients to be less affected by negativity. Whilst this time scale would be forensically more viable, no studies have attempted this in relation to facial composite construction.

The current study sought to address this deficiency by combining EvoFIT with a brief focused breathing technique. EvoFIT is a recognition-based facial construction system that employs interviewing techniques (e.g., cognitive interviewing mnemonics) shown to help enhance witness recall [7]. These techniques, assessed using a so called "Gold Standard" procedure [8], have been shown to improve composite accuracy [7, 22]. The current study involves this gold standard protocol to evaluate the potential benefit of a focused breathing instruction to relax witnesses, and thereby enhance witness recognition and recall; note that the focused breathing instruction is not the full mindfulness procedure, just one component of it [21]. We expect this instruction to relax witnesses and so improve both face recall and the effectiveness of their composite.

\section{Methods}

\subsection{Participants}

\subsubsection{Stage 1: Face Construction}

Participants were first year undergraduate Psychology students recruited by opportunity sampling from the University of Stirling. They were awarded course credit and a small cash incentive for participation. All had normal or corrected-to-normal vision. Target images were unfamiliar to participants (university staff who did not teach these first year students). The 20 participants were allocated to either the experimental (Focused Breathing) condition (9 females, 1 male) with an age range from 17 to 44 years old (Mage $=21$ years, $S D=8$ ) or to the control condition (8 females, 2 males) with an age range of 17 to 51 years old (Mage $=27$ years, $S D$ $=14$ ). Chance sampling effects led to a small increase in age in the control group relative to experimental (Mdiff $=6$ years) but this difference was not reliable, $t(18)=1.14, p=.27$.

\subsubsection{Stage 2: Composite Naming}

A total of 40 volunteer participants were an opportunity sample from available fourth year Psychology students and departmental staff (22 females, 18 males) with an age range from 20 to 55 years old (Mage $=24$ years, $S D=6)$. All had normal or corrected-to-normal vision. Participants were familiar with the target images as they had been taught by or were colleagues of those lecturers involved in Stage 1. All participants evaluated all composites.

\subsection{Materials}

The stimuli comprised images of 10 members of psychology department staff (five female). Images were colour full faced frontal photographs, clear and of neutral facial expression. Two sets of 10 colour photographs, $18 \mathrm{~cm}$ high by $13 \mathrm{~cm}$ wide, were printed on A4 paper for presentation to participants in Stage 1 . These were placed into numbered envelopes by a third person, so that the experimenter did not know the identity that would be seen by a given 'witness'.

Composites were constructed on a desktop personal computer using EvoFIT (v1.6.70) software. Two booklets were designed comprising a total of 14 composites from each condition; these were of the 10 target images (constructed by participants) and four 'foils' (unfamiliar composites not from the target images), and were placed in each booklet. A third booklet contained the ten original digital images of staff.

\subsection{Procedure}

The 'gold standard' for facial construction procedure [14] was followed. This entailed procedures that, as far as possible, should mirror the conditions witnesses would construct composites under police supervision. Firstly, target images should be unfamiliar to participants constructing the composites but familiar to those participants evaluating them. Secondly, the time delay between the participant seeing the target image and face construction should be a minimum of nominally 24 hours. Finally, a cognitive interview and a holistic-cognitive interview were used, as they have previously been shown to help with both face construction (recall) and evaluation (face-recognition) stages [14].

2.3.1. Construction Stage. Participants individually attended two separate sessions over consecutive days. On day one, they studied a target image for 1 minute, randomly selected from the set of 20 target images, and returned 20 to 28 hours later to construct a composite of the image from memory. Procedures were contingent on whether participants had been allocated to experimental or control group.

For the control group, procedures were as recommended to the police for use with EvoFIT, as described in detail in Fodarella et al. [14]. Here, participants freely recalled the appearance of the face and then reflected on the perceived personality of the face. For the experimental group, procedures were the same with the addition of a focused breathing instruction delivered prior to the cognitive interview. This was designed to help participants relax and involved guided focused breathing. During the cognitive and 
holistic-cognitive interviews, further focused breathing instructions (e.g., "close your eyes and take a focused breath while visualising the face") were given to participants. Conditions were counter-balanced across participants.

2.3.2. EvoFIT Construction Procedure. Detailed procedures for face construction using EvoFIT are described in [14]. In brief, participants selected an appropriately aged gender and age database. They then were asked to select from whole-face arrays, thought to be more relevant in familiar face recognition thus potentially helping to increase composite identification accuracy [4]. These witnesses were instructed to ignore the width of the face, as that aspect of the face could be finalised later, but to make selections based on the upper half of the face, since this procedure should promote an identifiable face. They selected items (presented as internal-features) for smooth faces, textured faces and combinations thereof. Once two complete iterations had been carried out, the witness manipulated the whole face for the overall or 'holistic' properties of the face (for age, weight, pleasantness and 12 other global scales) as well as adjusting the shape and position of features; when done, the external features (e.g., hair, ears, neck) were added. Following any further adjustments to holistic or shape properties, the face was saved to disk as the facial composite; see Figure 1 for examples. Participants in the experimental condition were asked to take a single focused breath prior to looking at ach EvoFIT screen. The cognitive interview and composite session took about an hour to complete per person.
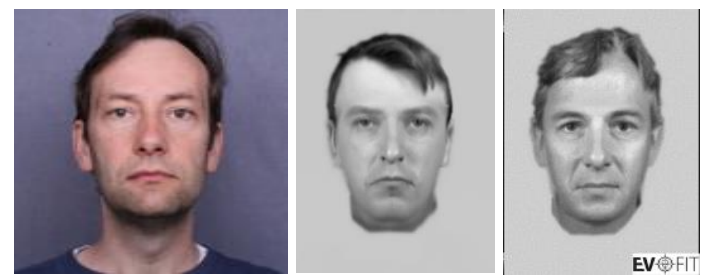

Figure 1. Example target and composites (left from a participant in the Control group and right from a participant in the focused breathing group).

2.3.3. Evaluation Stage Procedure. Participants were tested individually and informed that they would see several composites, some of which were lecturers in the Psychology Department with whom they should be familiar. Composites were separated into two booklets appropriate to experimental or control groups. Booklets contained 10 target images and four foils. Participants were informed that the 14 composites included an undisclosed number of foils. The use of foils in this way aimed to counter guessing or a simple process of elimination resulting from the relatively small population from which target images had been selected.

Composites were presented one at a time and participants were asked if they could identify the image. A correct name or definitive biographical information in lieu of a correct name (e.g., "he did the clinical lectures in the third year") was acceptable. Statements such as "he is a psychology lecturer" were not. Composites were presented sequentially in a different random order for each participant, from one booklet and then the other, with no time limit set; each composite remained visible until a response was given (a specific person or "don't know"). Participants were randomised to see one or other booklet first, counterbalanced. Afterwards, a third booklet containing the 10 target images was presented to participants to name (as a check that the targets were actually familiar to participants). The naming procedure took about 15 minutes per person.

\section{Results}

\subsection{Accurate Composite Identification}

Target images which were not correctly named were taken into account in the analyses, since a participant would not be able to correctly name a composite if that identity was unfamiliar to him or her. In this case, a conditional naming score was calculated based on the number of composites and targets that were correctly named. For example, if three composites and nine target images were correctly named, the naming score for this participant would be 3/9 (33.3\%).

A paired samples t-test was conducted to assess the effect of focused breathing instructions on the accuracy of composite identification. The one-tailed p-value is reported as the test was originally planned to be directional.

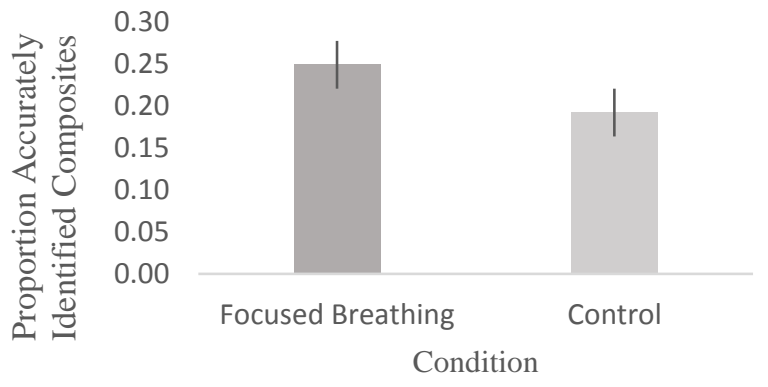

Figure 2. Mean and standard error of composite accuracy by condition.

Figure 2 shows the accuracy of composite naming in the two conditions. A paired t-test confirmed a significant benefit of composites from focused breathing $(M=0.25, S D=0.14)$ over control $(M=0.19, S D=0.15), t(39)=2.04, p=.020, d$ $=0.32$.

\subsection{Falsely Identified Composites}

A one-tailed paired samples t-test (selected due to the predictive of the effect) assessed the effect focused breathing instructions had on mistaken (inaccurate) names given to composites in the naming task. Figure 3 illustrates that focused breathing $(M=0.22, S D=0.17)$ produced somewhat fewer mistaken names to composites (an advantage) than 
control $(M=0.28, S D=0.20)$. A paired t-test found an approaching benefit for the focused breathing condition, $t(39)$ $=1.60, p=.06, d=0.25$.

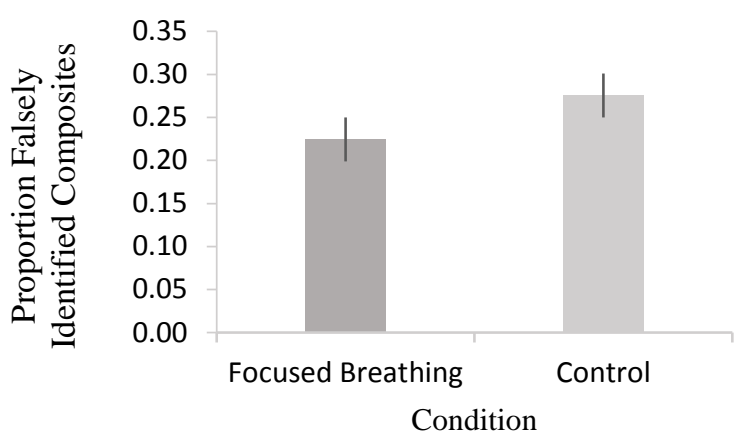

Figure 3. Mean and standard error of falsely identified composites by condition.

\subsection{Composite Construction Time}

Table 1 shows the mean composite construction time taken across both conditions. Focused breathing composites took slightly longer, and were slightly less variable (lower $S E$ ), but an independent samples t-test found no significant difference in composite construction time across conditions, $t(18)=$ $0.23, p=.82, d=0.10$.

Table 1. Composite Construction Time by Condition

\begin{tabular}{lcc}
\hline Condition & $\begin{array}{c}\text { Mean } \\
\text { (minutes) }\end{array}$ & $\begin{array}{c}\text { SE } \\
\text { (minutes) }\end{array}$ \\
\hline Focused Breathing & 65.5 & 4.0 \\
Control & 64.0 & 5.3 \\
\hline
\end{tabular}

\subsection{Total Number of Descriptive Words Used by Participants During Free Recall}

The experimenter and an additional researcher, with no prior knowledge of the aims of the study, calculated the total number of items recalled in each of the free recall sessions for participants in Stage 1. There was excellent internal consistency between the two raters on the total number of descriptive words used in the free recall sessions $(N=213$ words, $\alpha=.99$ ). This result provides confidence for the accuracy of data used in the following analysis.

Table 2 reveals that somewhat more words were recalled in total with focused breathing. Generalized Estimating Equations on the total recall score per participant (modelled with a Poisson log-linear function; Exchangeable WCM; and a model-based estimator) by group ( $0=$ Control and $1=$ Focused breathing) indicated that this difference approached significance, $X^{2}(1)=3.4, p=.07$, Odds Ratio $\operatorname{Exp}(B)=1.3$.
Table 2. Total number of descriptive words freely recalled by participants by condition

\begin{tabular}{lc}
\hline Condition & Total Descriptive Words \\
\hline Focused Breathing & 120 \\
Control & 93 \\
\hline
\end{tabular}

\section{Discussion}

Where use of a facial composite is important to a police investigation, witness memory of the offender is vital. Various techniques such as cognitive interviewing mnemonics are thus used to enhance witness performance. Here we looked to assess a mindfulness technique (focused breathing) when added to existing EvoFIT face construction procedures.

\subsection{Composite naming}

The naming task found significantly higher levels of correct naming for composites constructed by the focused breathing (cf. Control) group. Results indicate that this procedure will promote six additional correct names from every 100 crimes where EvoFIT composites would be used. Additionally, there was weak evidence of a reduction in the number of false (mistaken) names given under this manipulation. Any named composite should be investigated by police, including those incorrectly named. Investigating and interviewing innocent people is generally a waste of police time and resources [22] and so any technique that increases correct naming with no increase (or a reduction) in false identifications would be valuable. This current outcome would seem to be a worthwhile improvement for policing, a useful increase in the correct identification of offenders and a marginal reduction in the number of incorrect identifications.

Caution should be observed, however. Laboratory trials do not necessarily reflect real life situations and the true test of the current study can only really be determined by police using it in actual criminal investigations [10]. Hancock and colleagues suggested the number of arrests made from composites may be a good measure of how successful is a composite [10]. Their laboratory results $(26 \%)$ were similar to EvoFIT field trials (25-38\% [22]). The current study found similar results (25\% accuracy), which similarly demonstrate some compatibility between laboratory results and real life. However, other factors (e.g., witness anxiety and stress) can impact upon accuracy levels in real life settings.

\subsection{Stress}

No specific measure of stress was taken of participants in the study. However, the emotional impact of witnessing an actual crime is likely to far outweigh that experienced by first year 
psychology students taking part in a psychology experiment, one which rewarded them only in a small way for participation [23-24]. The literature is mixed on the effect of stress on eyewitness memory [25], but generally supports the notion that too much stress can adversely affect memory [10, 23]. This can be particularly relevant in highly stressful conditions [26], such as where a witness may be reluctant to discuss a crime (perhaps as he or she is also a victim), thus elevating stress levels beyond healthy limits [6]. Under these circumstances, it may be useful for police to have additional techniques such as those involved in mindfulness, to help relax witnesses and, in doing so, improve the effectiveness of their evidence.

\subsection{Focused Breathing and Recall vs. Recognition}

Whilst previous studies have demonstrated benefits of mindfulness in areas such as memory retrieval [18] and anxiety reduction [20], few have applied this technique to criminal investigation proceedings and witness recall. One notable exception is the focused breathing exercise included in the Liverpool Interview Protocol [27]. This protocol improved accurate recall from witnesses whilst showing no increase in mistaken identification [28]. Comparisons can be made with the current study. Higher levels of accurate information were found in the focused breathing group and marginally fewer inaccurate identifications. This would appear to support the efficacy and usefulness of a focused breathing instruction in a forensic setting. Also, analysis of the number of descriptive type adjectives used during the free recall sessions showed a higher number for the focused breathing group, a difference that approached significance. This may indicate an (albeit weak) effect on recall using the breathing instruction. A larger sample would be required to further assess the reliability of this DV as well as the DV for mistaken naming.

It is difficult to say if focused breathing improves recall, recognition or both. EvoFIT is a recognition based system which has been shown to be superior to feature-based and manual systems $[2,8,22]$. The application of the mindfulness instruction prior to cognitive and holistic-cognitive interviewing (recall aids) and the, albeit weak, association with descriptive words would suggest some benefit to recall mechanisms. Future research could apply the focused breathing instruction to witnesses prior to evaluating completed composites (primarily a recognition process). This may demonstrate a benefit more specifically related to recognition.

\subsection{Other Considerations}

There was little difference in the time taken to construct a composite in either condition. This seems to indicate that there is no loss of memory associated with the length of time taken to make choices during facial construction [28]. As the mean time to construct a composite was only 90 seconds longer for the focused breathing group, adding the instruction to current procedures should not unduly impact on police resources.

\subsection{Final comments}

The overall means across conditions was somewhat lower than current EvoFIT efficacy (60\% [2]). This may have resulted somewhat from operator inexperience [29]. EvoFIT training processes (www.evofit.com) recommend a five-day course and extensive practice (minimum of 20 composites constructed) for practitioners prior to conducting interviews and face constructions with witnesses. Due to time constraints, the experimenter received more limited training and completed 12 composites before the study commenced. This more limited experience may have somewhat reduced performance. The current procedure also included an instruction for witnesses to focus on the upper half of the face. This procedure was intended to reduce individual differences in witness performance and allow greater focus on the eye region. However, while achieving the former, the procedure may not be optimal since witnesses by be inclined to take into account the nose, which would be included in the upper half of the face (in the presented internal features region). The nose does not seem to be particularly important for later identification of a composite; a more recent procedure involves a direct request to witnesses to specifically select faces for the region around the eyes [30]. In conclusion, the focused breathing instruction would appear to be a useful addition to police practitioners who use EvoFIT, or indeed potentially for other types of holistic systems [3], at least for some witnesses. Our plan is to trial the technique in police interviews with witnesses and victims and solicit feedback on its apparent benefit and any operational difficulty in its administration.

\section{References}

[1] C.D. Frowd et al. "Whole-face procedures for recovering facial images from memory." Science and Justice, 2013, 53, pp. 89-97.

[2] C.D. Frowd, M. Pitchford, F. Skelton, A. Petkovic, C. Prosser $\&$ B. Coates, "Catching even more offenders with EvoFIT facial composites." Proceedings of 3rd International Conference on Emerging Security Technologies, 2012, pp. 20-26.

[3] C.D. Frowd, "Facial Composites and techniques to improve image recognisability." In T. Valentine, \& J. Davis (Eds.) Forensic Facial Identification: Theory and Practice of Identification from Eyewitnesses, Composites and Cctv, Wiley, 2015, pp. 43-70.

[4] V. Bruce, Recognising faces, Hove, Sussex, Lawrence Erlbaum Associates, 1988.

[5] C. Dando, R. Wilcock \& R. Milne, "The cognitive interview: Inexperienced police officers' perceptions of their witness/victim interviewing practices." Legal and Criminological Psychology, 2008, 13, pp. 59-70. 
[6] P. Risan, P-E. Binder \& R. Milne,"Regulating and coping with distress during police interviews of traumatized victims." Psychological Trauma: Theory, Research, Practice and Policy, 2016, 8, pp. 736-744.

[7] C.D. Frowd, L. Nelson, F. Skelton, R. Noyce, R. Atkins, P. Heard, et al. "Interviewing Techniques for Darwinian FacialComposite Systems." Applied Cognitive Psychology, 2012, 1, pp. $1-37$.

[8] R.E. Geiselman, R. Fisher, D. MacKinnon \& H. Holland, "Eyewitness Memory Enhancement in the Police Interview: Cognitive Retrieval Mnemonics Versus Hypnosis.” J Appl Psychol, 1985, 70, pp. 401-412.

[9] C.D. Frowd, V. Bruce, A.J. Smith \& P.J.B. Hancock, "Improving the quality of facial composites using a holistic cognitive interview." Journal of Experimental Psychology: Applied, 2008, 14, pp. 276-287.

[10] P.J.B. Hancock, K. Burke \& C.D. Frowd, C. D, "Testing facial composite construction under witness stress." International Journal of Bio-Science and Bio-Technology, 2011, 3, pp. 65-71.

[11] R.P. Fisher \& R.E. Geiselman, "The Cognitive Interview method of conducting police interviews: Eliciting extensive information and promoting Therapeutic Jurisprudence." International Journal of Law and Psychiatry, 2010, 33, pp. 321328 .

[12] M. Eysenck \& M. Calvo, "Anxiety and performance: The processing efficiency theory." Cognition and Emotion, 1992, 6, pp. $409-434$

[13] J.M Kieckhaefer, J.P. Vallano, \& N. Schreiber Compo, "Examining the positive effects of rapport building: When and why does rapport building benefit adult eyewitness memory?" Memory, 2014, 22, pp. 1010-1023.

[14] C. Fodarella, H. Kuivaniemi-Smith, J. Gawrylowicz \& C.D. Frowd, "Forensic Procedures for facial-composite construction." Journal of Forensic Practice, 2015, 17, pp. 259-270.

[15] A. Chiesa, R. Calati \& A. \& Serretti, "Does mindfulness training improve cognitive abilities? A systematic review of neuropsychological findings." Clinical Psychology Review, 2011, 31, pp. 449-464

[16] R.A. Baer, "Mindfulness training as a clinical intervention: A conceptual and empirical review." Clinical Psychology: Science and Practice, 2003, 10, pp. 125-143.

[17] R. Chambers, B.Y.C. Lo \& N.B. Allen, "The impact of intensive mindfulness training on attentional control, cognitive style, and affect." Cognitive Therapy and Research, 2008, 32, pp. 303-322.

[18] A.P. Jha, E.A. Stanley, A. Kiyonaga, L. Wong, \& L. Gelfand, "Examining the protective effects of mindfulness training on working memory capacity and affective experience". Emotion, 2010, 10, pp. 54-64.

[19] J. Kabat-Zinn "Mindfulness-based interventions in context: Past, present, and future." Clinical Psychology: Science and Practice, 2003, 10, pp. 144-156.

[20] J. Kabat-Zinn et al. "Effectiveness of a Meditation-Based Stress Reduction Program in the Treatment of Anxiety
Disorders.” American Journal of Psychiatry, 1992, 149, pp. 936943.

[21] J.J. Arch \& M.G. Craske, "Mechanisms of mindfulness: Emotion regulation following a focused breathing induction." Behaviour Research and Therapy, 20076, 44, pp. 1849-1858.

[22] C.D. Frowd, P.J.B. Hancock, V. Bruce, F. Skelton, C. Atherton, L. Nelson et al. "Catching More Offenders with EvoFIT Facial Composites: Lab Research and Police Field Trials." Global Journal of Human Social Science, 2011, 11, pp. $35-46$.

[23] K.A. Deffenbacher, B.H. Bornstein, S.D. Penrod, \& E.K. Mcgorty, "A Meta-Analytic Review of the Effects of High Stress on Eyewitness Memory." Law and Human Behavior, 2004, 28, pp. 687-706.

[24] C. Ihlebæk, T. Løve, D.E. Eilertsen \& S. Magnussen, "Memory for a staged criminal event witnessed live and on video." Memory, 2003, 11, pp. 319-327.

[25] J.C. Yuille \& J.L. Cutshall, "A case study of eyewitness memory of a crime." The Journal of Applied Psychology, 1986, 71, pp. 291-301.

[26] C.A. Morgan et al. "Accuracy of eyewitness memory for persons encountered during exposure to highly intense stress." International Journal of Law and Psychiatry, 2004, 27, pp. 265279.

[27] G.F. Wagstaff \& J.M Wheatcroft, Manual for the Liverpool Interview Protocol. Liverpool: Witness Research Group, University of Liverpool, 2012.

[28] G.F. Wagstaff, J.M. Wheatcroft, J. D. Hoyle \& C. Duffy, "Enhancing memory with the liverpool interview protocol: Is an association with hypnosis a problem?" Contemporary Hypnosis and Integrative Therapy, 2014, 30, pp. 142-151.

[29] C.D. Frowd, D. McQuiston-Surrett, S. Anandaciva, C.G. Ireland \& P.J.B. Hancock, "An evaluation of U.S. systems for facial composite production." Ergonomics, 2007, 50, pp. 19871998.

[30] C.D. Frowd, "Facial composite systems: Production of an identifiable face." In M. Bindemann and A. Megreya (Eds.) Face Processing: Systems, Disorders and Cultural Differences, 2017, Nova Science.

Due to an oversight we omitted two references from the original version:

[31] Fredman, M.D., Wheatcroft, J.M, \& Wagstaff, G.F. Breathe, describe, recognise: Improving eyewitness identification with a short focused breathing exercise. Poster presented to The European Association of Psychology and Law Conference, Nuremburg, 4-7 August 2015.

[32] Fredman, M.D., Wheatcroft, J.M. \& Wagstaff, G.F. Enhancing face recognition through a short focused meditation exercise. Poster presented to the Division of Forensic Psychology Annual Conference 2015, Manchester Metropolitan University, 1-3 July 2015 